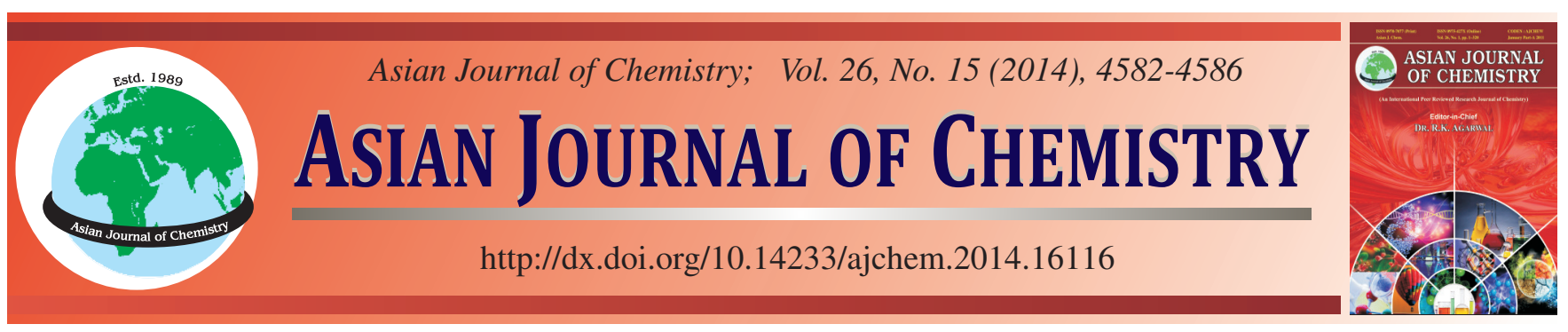

\title{
Anti-Oxidative and Anti-Inflammatory Effects of Phenolic Compounds from the Stems of Quercus acuta Thunberg
}

\author{
M.H. OH, K.H. PARK, M.H. KIM, H.H. KIM, S.R. KIM, K.J. PARK, J.H. HeO and M.W. LeE*
}

College of Pharmacy, Chung Ang University, Seoul 156-756, Republic of Korea

*Corresponding author: Fax: +82 2 8229778; Tel: +82 2 8205602; E-mail: mwlee@ cau.ac.kr

Two flavan 3-ols i.e., (+)-catechin (1), (-)-epicatechin (2)], three flavanonols i.e., [taxifolin (3), taxifolin 3-O- $\beta$-D-glucopyranoside (4) and taxifolin 4'-O- $\beta$-D-glucopyranoside (5), one proanthocyanidin i.e., procyanidin B-3 (6) and one i.e., neolignan (+)-lyoniresinol 3 $\alpha-O-\beta$ D-xylopyranoside (7) have been isolated from the stems of Quercus acuta Thunberg (QAS) by anti-oxidative activity-guided isolation. Although these compounds have been reported previously. To our best of knowledge, this is the first study to report their isolation from Q. acuta Thunberg. Anti-oxidative activity was measured using the 2,2-diphenyl-1-picrylhydrazyl (DPPH) free radical and superoxide dismutase (SOD)-like superoxide anion (generated by xanthine oxidase/hypoxanthine) scavenging assays. Anti-inflammatory activity was assessed by quantifying the nitric oxide in the supernatant of lipopolysaccharide (LPS)-stimulated mouse Raw 264.7 macrophage cells.

Keywords: Quercus acuta, Phenolic compound, DPPH, Xanthine oxidase, Nitric oxide.

\section{INTRODUCTION}

Approximately, 200 species of Quercus grow in the temperate regions of the northern hemisphere ${ }^{1}$. The 5 indigenous species of Quercus found in Korea include Quercus acuta Thunberg, $Q$. gilva Blume, $Q$. glauca Thunberg, $Q$. myrsinaefolia Blume and $Q$. salicina Blume. $Q$. acuta Thunberg is native to both Japan and Korea ${ }^{2}$. Phytochemical investigations of plants belonging to the Quercus genus have revealed the presence of phenolic glucosidic gallates ${ }^{3,4}$ and tannins ${ }^{5,6}$. Gallic acid and protoquercitol gallates, namely, 4,5di-O-galloyl (+)-protoquercitol and 3,5-di-O-galloyl (+)-protoquercitol, were isolated from $Q$. acuta. Evaluation of biological activity of $Q$. acuta has revealed its antibacterial effect ${ }^{7}$. The presence of phenolic compounds in $Q$. acuta has not been reported. In this study, we evaluated the anti-oxidative and anti-inflammatory effects of compounds from the stems of Q. acuta.

\section{EXPERIMENTAL}

Stationary phases for column chromatographic isolation were Sephadex LH-20 (10-25 $\mu \mathrm{m}$; GE Healthcare Bio-Science AB, Uppsala, Sweden), MCI-gel CHP 20P (75-150 $\mu \mathrm{m}$; Mitsubishi Chemical, Tokyo, Japan) and ODS-B gel (40$60 \mu \mathrm{m}$; Daiso, Osaka, Japan). ODS-B gel was used as the stationary phase in middle pressure liquid chromatography
(MPLC) analysis. Thin layer chromatography (TLC) was carried out with a precoated silica gel plate $\left(60 \mathrm{~F}_{254}\right.$; Merck, Darmstadt, Germany) with the mobile phase being chloroform, methanol and water in the ratio of 6:4:1. The spots were detected under UV radiation ( $254 \mathrm{~nm}$ ) after spraying the plate with ferric chloride $\left(\mathrm{FeCl}_{3}\right)$ and $10 \%$ sulfuric acid $\left(\mathrm{H}_{2} \mathrm{SO}_{4}\right)$ or anisaldehyde- $\mathrm{H}_{2} \mathrm{SO}_{4}$ followed by heating.

The compounds present in $Q$. acuta were identified by several instrumental analyses. One-dimensional nuclear magnetic resonance (NMR; ${ }^{1} \mathrm{H}-(300$ or $600 \mathrm{MHz})$ and ${ }^{13} \mathrm{C}-(75$ or $125 \mathrm{MHz}$ ) NMR) and 2D-NMR (proton, proton correlation spectroscopy (HH-COSY), heteronuclear single quantum coherence (HSQC) and heteronuclear multiple bond coherence (HMBC)) were recorded with Gemini 2000 and VNS (Varian, Palo Alto, CA, USA) at the center for research facilities of Chung-Ang University. Low-resolution fast atom bombardment mass spectrum (LR-FAB-MS) was recorded using JMSAX505WA (JEOL, Tokyo, Japan) at the National Center for Inter-University Research Facilities of Seoul National University. Optical rotation was recorded with Autopol III (Rudolph Research, Hackettstown, NJ, USA) at the National Instrumentation Center for Environmental Management at Seoul National University.

The stems of $Q$. acuta $(6.5 \mathrm{~kg})$ were obtained from Halla Arboretum on Jeju Island (Korea) in April 2009. A voucher 
specimen (NO.171) was deposited at the herbarium of College of Pharmacy, Chung-Ang University.

Extraction and isolation: The stems of $Q$. acuta $(6.5 \mathrm{~kg})$ were extracted several times with $80 \%$ acetone at room temperature. Concentrating the acetone extract under vacuum yielded $430 \mathrm{~g}$ of the extract. Q. acuta constituents were isolated by solvent fractionation with hexane, ethyl acetate, butyl alcohol and water. The ethyl acetate and butyl alcohol layers ( 81 and $130 \mathrm{~g}$, respectively) showed antioxidant activity corresponding to $\mathrm{IC}_{50}$ values of $18.09 \pm 1.53$ and $20.67 \pm 2.21 \mu \mathrm{g} / \mathrm{mL}$, respectively, with $\mathrm{IC}_{50}$ of L-ascorbic acid positive control being 4.29 $\pm 0.08 \mu \mathrm{g} / \mathrm{mL}$ (data not shown). The butyl alcohol layer applied to the Sephadex LH-20 column $(25-100 \mu \mathrm{m} ; 2000 \mathrm{~g} ; 10 \times 120$ $\mathrm{cm})$ and eluted with water and methanol yielded 8 subfractions. Subfraction 6 was further separated on an ODS-B gel (50 $\mu$ m; $400 \mathrm{~g} ; 3 \times 50 \mathrm{~cm}$ ) with $20-100 \%$ methanol in an MPLC system $(5 \mathrm{~mL} / \mathrm{min} ; 280 \mathrm{~nm})$ and Sephadex LH-20 (25-100 $\mu \mathrm{m} ; 300 \mathrm{~g}$; $2.5 \times 40 \mathrm{~cm} ; 10-100 \%$ ethanol gradient system) to yield (+)catechin $(\mathbf{1}, 370 \mathrm{mg})$ and (-)-epicatechin $(2,43 \mathrm{mg})$.

The ethyl acetate layer applied to the Sephadex LH-20 column $(25-100 \mu \mathrm{m} ; 2000 \mathrm{~g} ; 10 \times 120 \mathrm{~cm})$ and eluted with $\mathrm{H}_{2} \mathrm{O}-\mathrm{MeOH}$ resulted in 10 subfractions. Subfraction 6 was separated on ODS-B gels $(50 \mu \mathrm{m}, 300 \mathrm{~g} ; 3.5 \times 60 \mathrm{~cm}),(50$ $\mu \mathrm{m} ; 250 \mathrm{~g} ; 3 \times 50 \mathrm{~cm})$ and $(50 \mu \mathrm{m}, 150 \mathrm{~g} ; 1.5 \times 45 \mathrm{~cm})$ with a methanol gradient $\left(\mathrm{H}_{2} \mathrm{O}-100,20-100\right.$ and $\left.30-100 \%\right)$ in an MPLC system $(5 \mathrm{~mL} / \mathrm{min} ; 280 \mathrm{~nm})$ to obtain taxifolin $3-O-\beta$ D-glucopyranoside $(4,105 \mathrm{mg})$ and taxifolin $4 '-O-\beta$-Dglucopyranoside $(\mathbf{5}, 310 \mathrm{mg})$. Subfraction 8 (3.75 g) was further separated on an ODS-B gel $(50 \mu \mathrm{m} ; 300 \mathrm{~g} ; 3 \times 40 \mathrm{~cm})$ with 20-100\% methanol gradient in an MPLC system $(5 \mathrm{~mL} /$ $\min ; 280 \mathrm{~nm})$ and Sephadex LH-20 (25-100 $\mu \mathrm{m} ; 200 \mathrm{~g} ; 2.5 \times$ $30 \mathrm{~cm} ; 100-10 \%$ ethanol gradient system) to obtain procyanidin B-3 (6, $51 \mathrm{mg})$ and taxifolin $(\mathbf{3}, 101 \mathrm{mg})$. Subfraction 3 $(1.89 \mathrm{~g})$ was separated on an ODS-B gel $(50 \mu \mathrm{m} ; 200 \mathrm{~g} ; 2.5 \times$ $30 \mathrm{~cm}$ ) with 10-100\% methanol gradient in an MPLC system $(5 \mathrm{~mL} / \mathrm{min} ; 280 \mathrm{~nm})$ and recrystallization of subfraction 3 yielded (+)-lyoniresinol 3a- $O$ - $\beta$-D-xylopyranoside $(7,250 \mathrm{mg})$

(+)-Catechin (1): White amorphous powder; EI-MS: $m / z 290[\mathrm{M}]^{+} ;{ }^{1} \mathrm{H}$ NMR $\left(300 \mathrm{MHz}, \mathrm{MeOH}-d_{4}+\mathrm{D}_{2} \mathrm{O}\right): \delta 2.50$ $\left(1 \mathrm{H}, \mathrm{dd}, J=8.4,16.0 \mathrm{~Hz}, \mathrm{H}-4_{\mathrm{ax}}\right), 2.84(1 \mathrm{H}, \mathrm{dd}, J=5.7,16.0$ $\left.\mathrm{Hz}, \mathrm{H}-4_{\mathrm{eq}}\right), 3.97$ (1H, m, H-3), 4.55 (1H, d, J = 7.5 Hz, H-2), $5.85(1 \mathrm{H}, \mathrm{d}, J=2.1 \mathrm{~Hz}, \mathrm{H}-6), 5.92(1 \mathrm{H}, \mathrm{d}, J=2.1 \mathrm{~Hz}, \mathrm{H}-8)$, $6.71\left(1 \mathrm{H}, \mathrm{dd}, J=1.6,8.1 \mathrm{~Hz}, \mathrm{H}-6{ }^{\prime}\right), 6.76(1 \mathrm{H}, \mathrm{d}, J=8.1 \mathrm{~Hz}$, H-5') and $6.83\left(1 \mathrm{H}, \mathrm{d}, J=1.6 \mathrm{~Hz}, \mathrm{H}-2^{\prime}\right)$.

${ }^{13} \mathrm{C}$ NMR $\left(75 \mathrm{MHz}, \mathrm{MeOH}-d_{4}+\mathrm{D}_{2} \mathrm{O}\right): \delta 28.5(\mathrm{C}-4), 68.8$ (C-3), 82.9 (C-2), 95.5 (C-8), 96.3 (C-6), 100.8 (C-10), 115.3 (C-2'), 116.1 (C-5'), 120.1 (C-6'), 132.2 (C-1'), 146.3 (C-3', 4'), 157.6 (C-9), 157.8 (C-5) and 157.9 (C-7).

(-)-Epicatechin (2): White amorphous powder; EI-MS: $m / z 290[\mathrm{M}]^{+} ;{ }^{1} \mathrm{H}$ NMR (300 MHz, MeOH- $\left.d_{4}+\mathrm{D}_{2} \mathrm{O}\right): \delta 2.73$ $\left(1 \mathrm{H}, \mathrm{dd}, J=2.7,16.8 \mathrm{~Hz}, \mathrm{H}-4_{\mathrm{ax}}\right), 2.86(1 \mathrm{H}, \mathrm{dd}, J=4.8,16.8$ $\left.\mathrm{Hz}, \mathrm{H}-4_{\text {eq }}\right), 4.17$ (1H, br s, H-3), 4.81(1H, br s, H-2), $5.92(1 \mathrm{H}$, $\mathrm{d}, J=2.4 \mathrm{~Hz}, \mathrm{H}-6), 5.94(1 \mathrm{H}, \mathrm{d}, J=2.4 \mathrm{~Hz}, \mathrm{H}-8), 6.80(1 \mathrm{H}$, dd, $\left.J=1.5,8.1 \mathrm{~Hz}, \mathrm{H}-6^{\prime}\right), 6.75$ (1H, d, $\left.J=8.1 \mathrm{~Hz}, \mathrm{H}-5 '\right), 6.97$ $\left(1 \mathrm{H}, \mathrm{d}, J=1.5 \mathrm{~Hz}, \mathrm{H}-2^{\prime}\right)$.

${ }^{13} \mathrm{C}$ NMR $\left(75 \mathrm{MHz}, \mathrm{MeOH}-d_{4}+\mathrm{D}_{2} \mathrm{O}\right): \delta 29.3(\mathrm{C}-4), 67.5$ (C-3), 79.9 (C-2), 96.4 (C-6, 8), 100.1 (C-10), 115.3 (C-2'), $115.9\left(\mathrm{C}^{\prime} 5^{\prime}\right), 119.4\left(\mathrm{C}^{-6} \mathbf{6}^{\prime}\right), 132.3\left(\mathrm{C}-1^{\prime}\right), 145.8$ (C-3', $\left.4^{\prime}\right), 157.6$ (C-9), $158.0(\mathrm{C}-5,7)$.
Taxifolin (3): White yellow amorphous powder; EI-MS: $m / z 304[\mathrm{M}]^{+} ;{ }^{1} \mathrm{H}$ NMR $\left(600 \mathrm{MHz}, \mathrm{DMSO}-d_{6}+\mathrm{D}_{2} \mathrm{O}\right): \delta 4.45$ $(1 \mathrm{H}, \mathrm{d}, J=11.1 \mathrm{~Hz}, \mathrm{H}-3), 4.93(1 \mathrm{H}, \mathrm{d}, J=11.1 \mathrm{~Hz}, \mathrm{H}-2), 5.82$ $(1 \mathrm{H}, \mathrm{d}, J=2.1 \mathrm{~Hz}, \mathrm{H}-6), 5.87(1 \mathrm{H}, \mathrm{d}, J=2.1 \mathrm{~Hz}, \mathrm{H}-8), 6.72$ $\left(2 \mathrm{H}, \mathrm{m}, \mathrm{H}-5{ }^{\prime}, 6^{\prime}\right)$ and $6.85\left(1 \mathrm{H}, \mathrm{d}, J=2.1 \mathrm{~Hz}, \mathrm{H}-2^{\prime}\right)$.

${ }^{13} \mathrm{C}$ NMR $\left(150 \mathrm{MHz}\right.$, DMSO- $\left.d_{6}+\mathrm{D}_{2} \mathrm{O}\right): \delta 71.8(\mathrm{C}-3), 83.4$ (C-2), 95.4 (C-8), 96.4 (C-6), 100.6 (C-10), 115.5 (C-2'), 115.6 (C-5'), 119.9 (C-6'), 128.4 (C-1'), 145.1 (C-4') 146.0 (C-3'), 162.9 (C-5, 9), 167.4 (C-7) and 197.8 (C-4).

Taxifolin 3- $\boldsymbol{O}$ - $\boldsymbol{\beta}$-D-glucopyranoside (4): Brown amorphous powder; LR-FAB-MS: $m / z 465$ [M-H] ; ${ }^{1} \mathrm{H}$ NMR $(300 \mathrm{MHz}$, $\left.\mathrm{MeOH}-d_{4}+\mathrm{D}_{2} \mathrm{O}\right): \delta 3.00(1 \mathrm{H}, \mathrm{m}$, glc-5), $3.11(1 \mathrm{H}, \mathrm{t}, J=9.0$ $\mathrm{Hz}$, glc-3), $3.21(1 \mathrm{H}, \mathrm{m}$, glc-2), $3.22(1 \mathrm{H}, \mathrm{t}, J=9.0 \mathrm{~Hz}$, glc-4), $3.60(1 \mathrm{H}, \mathrm{dd}, J=5.7,12.0 \mathrm{~Hz}$, glc-6a), $3.77(1 \mathrm{H}, \mathrm{dd}, J=1.8$, $12.0 \mathrm{~Hz}$, glc-6b), $3.85(1 \mathrm{H}, \mathrm{d}, J=7.5 \mathrm{~Hz}$, glc- 1$), 4.92(1 \mathrm{H}, \mathrm{d}$, $J=9.9 \mathrm{~Hz}, \mathrm{H}-3), 5.22(1 \mathrm{H}, \mathrm{d}, J=9.9 \mathrm{~Hz}, \mathrm{H}-2), 5.89(2 \mathrm{H}, \mathrm{m}$, H-6, 8), 6.77 (1H, d, $J=8.4$ Hz, H-5'), $6.83(1 \mathrm{H}, \mathrm{dd}, J=1.8$. $\left.8.4 \mathrm{~Hz}, \mathrm{H}-6^{\prime}\right)$ and $6.95\left(1 \mathrm{H}, \mathrm{d}, J=1.8 \mathrm{~Hz}, \mathrm{H}-2^{\prime}\right)$.

${ }^{13} \mathrm{C}$ NMR (75 MHz, DMSO- $\left.d_{6}+\mathrm{D}_{2} \mathrm{O}\right): \delta 62.7$ (glc-6), 71.3 (glc-4), 74.7 (glc-2), 77.2 (C-3), 77.7 (glc-5), 78.3 (glc-3), 83.6 (C-2), 96.4 (C-6), 97.2 (C-8), 102.7 (C-10, glc-1), 115.9 (C-2'), 116.2 (C-5'), 121.1 (C-6'), 129.0 (C-1'), 146.4 (C-3'), 147.4 (C-4'), 164.2 (C-9), 165.5 (C-5), 169.1 (C-7) and 196.0 (C-4).

Taxifolin 4'- $\boldsymbol{O}$ - $\boldsymbol{\beta}$-D-glucopyranoside (5): Brown amorphous powder; LR- FAB-MS: $m / z 465$ [M-H] ; ${ }^{1} \mathrm{H}$ NMR (300 $\mathrm{MHz}, \mathrm{MeOH}-d_{4}+\mathrm{D}_{2} \mathrm{O}$ ): $\delta 3.10-3.21$ (4H in total, m, glc-2, 3, 4, 5), $3.57(1 \mathrm{H}, \mathrm{dd}, J=3.9,11.7 \mathrm{~Hz}$, glc-6a), $3.80(1 \mathrm{H}, \mathrm{dd}, J=$ 1.2, $11.7 \mathrm{~Hz}$, glc-6b), $4.67(1 \mathrm{H}, \mathrm{d}, J=7.8 \mathrm{~Hz}$, glc- 1$), 4.88$ $(1 \mathrm{H}, \mathrm{d}, J=9.0 \mathrm{~Hz}, \mathrm{H}-3), 5.24(1 \mathrm{H}, \mathrm{d}, J=9.0 \mathrm{~Hz}, \mathrm{H}-3), 5.89$ $(2 \mathrm{H}, \mathrm{s}, \mathrm{H}-6,8), 6.75$ (1H, d, $J=8.1 \mathrm{~Hz}, \mathrm{H}-5$ '), 6.80 (1H, dd, $J$ $\left.=1.8 .8 .1 \mathrm{~Hz}, \mathrm{H}-6^{\prime}\right)$ and $6.96\left(1 \mathrm{H}, \mathrm{d}, J=1.8 \mathrm{~Hz}, \mathrm{H}-2^{\prime}\right)$.

${ }^{13} \mathrm{C}$ NMR $\left(75 \mathrm{MHz}, \mathrm{DMSO}-d_{6}+\mathrm{D}_{2} \mathrm{O}\right): \delta 62.9(\mathrm{glc}-6), 71.5$ (glc-4), 72.4 (C-3) 75.4 (glc-2), 77.7 (glc-3), 78.0 (glc-5), 83.4 (C-2), 95.4 (C-8), 97.3 (C-6), 102.4 (C-10), 104.6(glc-1), 116.0 (C-2'), 116.3 (C-5'), 121.2 (C-6'), 128.9 (C-1'), 146.0 (C-3'), 147.0 (C-4'), 164.1 (C-9), 165.6 (C-7), 169.1 (C-5) and 196.2 (C-4).

Procyanidin B-3 (6): Brown amorphous powder; LRFAB-MS: $m / z 577$ [M-H] ; ${ }^{1} \mathrm{H}$ NMR $\left(600 \mathrm{MHz}\right.$, DMSO- $d_{6}+$ $\left.\mathrm{D}_{2} \mathrm{O}\right): \delta 2.48\left(1 \mathrm{H}, \mathrm{dd}, J=8.4,16.2 \mathrm{~Hz}, \mathrm{H}-4 \mathrm{t}_{\mathrm{ax}}\right), 2.75(1 \mathrm{H}, \mathrm{dd}$, $\left.J=8.4,16.2 \mathrm{~Hz}, \mathrm{H}-4 \mathrm{t}_{\mathrm{eq}}\right), 3.78(1 \mathrm{H}, \mathrm{m}, \mathrm{H}-3 \mathrm{t}), 4.25(1 \mathrm{H}, \mathrm{d}, J=$ $10.2 \mathrm{~Hz}, \mathrm{H}-4 \mathrm{u}), 4.35(1 \mathrm{H}, \mathrm{t}, J=10.2 \mathrm{~Hz}, \mathrm{H}-3 \mathrm{u}), 4.40(1 \mathrm{H}, \mathrm{d}$, $J=8.4 \mathrm{~Hz}, \mathrm{H}-2 \mathrm{u}), 4.53(1 \mathrm{H}, \mathrm{d}, J=7.2 \mathrm{~Hz}, \mathrm{H}-2 \mathrm{t}), 5.78(1 \mathrm{H}, \mathrm{d}$, $J=1.2 \mathrm{~Hz}, \mathrm{H}-6 \mathrm{u}), 5.88(1 \mathrm{H}, \mathrm{d}, J=1.2 \mathrm{~Hz}, \mathrm{H}-8 \mathrm{u}), 6.06(1 \mathrm{H}, \mathrm{s}$, H-6t), 6.24 (1H, dd, $J=1.8,8.4 \mathrm{~Hz}, \mathrm{H}-6 \mathrm{t}$ ), 6.47 (1H, dd, $J=$ 2.2, 8.4 Hz, H-6'u), $6.58(1 \mathrm{H}, \mathrm{d}, J=1.8 \mathrm{~Hz}, \mathrm{H}-2 \mathrm{t}), 6.67(2 \mathrm{H}$, $\mathrm{d}, J=8.4 \mathrm{~Hz}, \mathrm{H}-5$ 'u, H-5't) and $6.73(1 \mathrm{H}, \mathrm{d}, J=2.4 \mathrm{~Hz}, \mathrm{H}-$ 2'u).

${ }^{13} \mathrm{C}$ NMR (150 MHz, DMSO- $\left.d_{6}+\mathrm{D}_{2} \mathrm{O}\right): \delta 27.3(\mathrm{C}-4 \mathrm{t}), 37.1$ (C-4u), $67.4(\mathrm{C}-3 \mathrm{t}), 72.2(\mathrm{C}-3 \mathrm{u}), 81.0(\mathrm{C}-2 \mathrm{t}), 82.5(\mathrm{C}-2 \mathrm{u}), 94.6$ (C-8u), 95.4 (C-6u, 6t), 100.8 (C-10t), 105.7 (C-10u), 106.7 (C-8t), 114.0 (C-2't), $114.6(\mathrm{C}-2$ 'u $), 114.7(\mathrm{C}-5$ 't $), 115.0$ (C5'u), 118.4 (C-6'u), 119.1 (C-6't), 130.4 (C-1'u), 131.1 (C-1't), 144.0 (C-3'u), 144.1 (C-4'u), 144.3 (C-3't), 144.6 (C-4't), 153.4 (C-9t), 154.2 (C-9u), $154.4(\mathrm{C}-5 \mathrm{t}), 155.6(\mathrm{C}-5 \mathrm{u}, 7 \mathrm{t})$ and 157.2 (C-7u).

(+)-Lyoniresinol 3 $\alpha-O$ - $\beta$-D-xylopyranoside (7): White amorphous powder; LR- FAB-MS: $m / z, 551$ [M-H] ; ${ }^{1} \mathrm{H}$ NMR 
$\left(600 \mathrm{MHz}, \mathrm{DMSO}-d_{6}+\mathrm{D}_{2} \mathrm{O}\right): \delta 1.56(1 \mathrm{H}, \mathrm{m}, \mathrm{H}-2), 1.99(1 \mathrm{H}$, m, H-3), $2.55\left(1 \mathrm{H}, \mathrm{dd}, J=-, 15.6 \mathrm{~Hz}, \mathrm{H}-1_{\mathrm{eq}}\right), 2.68(1 \mathrm{H}, \mathrm{dd}, \mathrm{J}=$ $\left.4.2,15.6 \mathrm{~Hz}, \mathrm{H}-1_{\mathrm{ax}}\right), 3.08$ ( $\left.1 \mathrm{H}, \mathrm{dd}, J=7.8,8.4 \mathrm{~Hz}, \mathrm{xyl}-2\right), 3.09$ $(1 \mathrm{H}, \mathrm{dd}, J=10.8,11.4 \mathrm{~Hz}, \mathrm{xyl}-5 \mathrm{~b}), 3.19(1 \mathrm{H}, \mathrm{t}, J=9.0 \mathrm{~Hz}$, xyl-3), $3.31\left(3 \mathrm{H}, \mathrm{s}, 5-\mathrm{OCH}_{3}\right), 3.32(1 \mathrm{H}, \mathrm{d}, J=3.6 \mathrm{~Hz}, \mathrm{H}-3 \alpha-$ b), 3.35 (1H, m, xyl-4), 3.40 (1H, dd, $J=6.9,10.8 \mathrm{~Hz}, \mathrm{H}-2 \alpha-$ b), 3.54 (1H, dd, $J=3.9,10.8 \mathrm{~Hz}, \mathrm{H}-2 \alpha-\mathrm{a}), 3.68$ (1H, m, H$3 \alpha-\mathrm{a}), 3.68\left(3 \mathrm{H}, \mathrm{s}, 5^{\prime}-\mathrm{OCH}_{3}\right), 3.69\left(3 \mathrm{H}, \mathrm{s}, 3^{\prime}-\mathrm{OCH}_{3}\right), 3.74(1 \mathrm{H}$, $\mathrm{dd}, J=5.4,11.4 \mathrm{~Hz}$, xyl-5a), $3.82\left(3 \mathrm{H}, \mathrm{s}, 7-\mathrm{OCH}_{3}\right), 4.18(1 \mathrm{H}$, $\mathrm{d}, J=7.8 \mathrm{~Hz}, \mathrm{xyl}-1), 4.31(1 \mathrm{H}, \mathrm{d}, J=6.6 \mathrm{~Hz}, \mathrm{H}-4), 6.38(2 \mathrm{H}$, s, H-2', 6') and 6.59 (1H, s, H-8).

${ }^{13} \mathrm{C}-\mathrm{NMR}\left(150 \mathrm{MHz}, \mathrm{DMSO}-d_{6}+\mathrm{D}_{2} \mathrm{O}\right): \delta 34.9(\mathrm{C}-1)$, 39.7 (C-2), 41.3 (C-4) $44.9(\mathrm{C}-3), 56.0\left(5-\mathrm{OCH}_{3}\right), 56.4\left(3^{\prime}, 5^{\prime}-\right.$ $\left.\mathrm{OCH}_{3}\right), 59.1\left(7-\mathrm{OCH}_{3}\right), 64.0(\mathrm{C}-2 \alpha), 66.0$ (xyl-5), 69.8 (xyl4), 69.5 (C-3 $\alpha$ ), 73.5 (xyl-2), 76.9 (xyl-3), 104.3 (xyl-1), 106.2 (C-2', 6'), 107.0 (C-8), 125.2 (C-10), 128.8 (C-9), 133.5 (C4'), 137.5 (C-6), 138.0 (C-1'), 146.8 (C-5), 147.2 (C-7) and $147.8\left(\mathrm{C}-3^{\prime}, 5^{\prime}\right)$.

\section{Biological assay}

DPPH radical scavenging activity: Antioxidative activity was measured by evaluating 1,1-diphenyl-2-picrylhydrzyl (DPPH) (Sigma, St. Louis, USA) scavenging activities ${ }^{8}$. Briefly, $20 \mu \mathrm{l}$ of the compounds was mixed with absolute ethanol and added to $180 \mu \mathrm{L}$ of DPPH solution $(0.1 \mathrm{mM}$ in absolute ethanol). After gentle mixing, the reactions were allowed to stand for $0.5 \mathrm{~h}$ and the optical densities were measured at $492 \mathrm{~nm}$ by using an enzyme linked immunosorbent assay (ELISA) reader (TECAN, Salzburg, Austria). Free radical scavenging activities were calculated as [(1-(sample optical density $(\mathrm{OD}) /$ control OD) $) \times 100]$ and were expressed as $\mathrm{IC}_{50}$ values, which were defined as the concentrations at which $50 \%$ of DPPH free radicals were scavenged. L-ascorbic acid was used as a positive control.

Superoxide anion scavenging activity: A reaction mixture was prepared with $50 \mathrm{mM}$ of phosphate buffer $(\mathrm{pH} 7.5)$ containing EDTA (0.05 mM), hypoxanthine $(0.2 \mathrm{mM}), 63 \mu \mathrm{L}$ of NBT [nitroblue-tetrazolium] (1 mM) (Sigma, St. Louis, MO), $63 \mu \mathrm{L}$ of aqueous or ethanolic extract (distilled water for the control) and $63 \mu \mathrm{L}$ of xanthine oxidase (1.2 unit/ $\mu \mathrm{L})$ (Sigma, St. Louis, MO) in an Eppendorf tube with the final volume being $632 \mu \mathrm{L}$. Xanthine oxidase was added last. For each sample, a blank solution devoid of the drug was prepared. The rate of NBT reduction was determined on the basis of sequential spectrophotometric determination of absorbance at $590 \mathrm{~nm}$. The solutions were freshly prepared each day and kept away from light. Results are expressed as the percentage inhibition of NBT reduction with respect to the reaction mixture without sample (buffer only). Superoxide anion scavenging activities were calculated as [(1-(sample OD-blank OD)/ $($ control OD-blank OD) $) \times 100]$ and were expressed as $\mathrm{IC}_{50}$ values, which were defined as the concentrations at which $50 \%$ of NBT/superoxide anions were scavenged ${ }^{9}$. Allopurinol (Sigma, St. Louis, MO) was used as a positive control.

Cell culture: RAW 264.7 cells were purchased from the Korean Cell Line Bank. These cells were grown at $37^{\circ} \mathrm{C}$ in a humidified atmosphere $\left(5 \% \mathrm{CO}_{2}\right)$ in DMEM medium (Sigma, St. Louis, MO, USA) containing $10 \%$ fetal bovine serum, $100 \mathrm{IU} / \mathrm{mL}$ penicillin $\mathrm{G}$ and $100 \mathrm{mg} / \mathrm{mL}$ streptomycin (Gibco
BRL, Grand Island, NY, USA) ${ }^{10}$. Cell enumeration was performed with the help of a hemocytometer.

Inhibitory activity on NO production: RAW 264.7 macrophage cells were cultured in a 96-well plate and incubated for $2 \mathrm{~h}$ at $37^{\circ} \mathrm{C}$ in a humidified atmosphere $\left(5 \% \mathrm{CO}_{2}\right)$. The cells were then incubated in a medium containing $0.1 \mu \mathrm{g}$ / mL LPS (Sigma, St. Louis, MO, USA) and the drug isolates. After incubating for an additional $24 \mathrm{~h}$, the NO content was analyzed by Griess assay. The Griess reagent $(0.1 \%$ naphthylethylenediamine and $1 \%$ sulfanilamide in $5 \%$ phosphoric acid solution) (Sigma, St. Louis, MO, USA) was added to the supernatant obtained from drug-treated samples. L-NMMA was used as a positive control. NO content was then read at $540 \mathrm{~nm}$ against a standard sodium nitrite curve. Inhibitory activity on NO production was calculated as inhibition rate $(\%)=[1-($ sample OD - blank OD $) /($ control OD - blank OD $)]$ $\times 100$ and $\mathrm{IC}_{50}$ values, which were defined as the concentration that could inhibit $50 \%$ of NO production ${ }^{11}$, was determined.

Cell cytotoxicity assay: Before performing the biological assay, cytotoxicity was measured by mitochondrial-dependent reduction of MTT [3-(4,5-dimethylthiazol-2-yl)-2,5-diphenyltetrazolium-bromide] (Sigma, St. Louis, MO, USA) to formazan ${ }^{11}$. After the cells were cultured in a 96-well plate and incubated for $2 \mathrm{~h}$, they were treated with the drug isolates $(12.5,25,50$ and $100 \mu \mathrm{g} / \mathrm{mL}$ or $\mu \mathrm{M})$. The cells were incubated for an additional $24 \mathrm{~h}$ and the medium was replaced with fresh medium contained $0.5 \mathrm{mg} / \mathrm{mL}$ MTT; the incubation was further continued for $4 \mathrm{~h}$ at $37^{\circ} \mathrm{C}$. The medium was then removed and the MTT-formazan complex thus formed was dissolved in $200 \mu \mathrm{L}$ of dimethylsulfoxide (DMSO). The extent of MTT reduction to formazan within the cells was quantified by measuring the absorbance at $540 \mathrm{~nm}$ by using an ELISA reader (TECAN, Salzburg, Austria) ${ }^{12}$. Cytotoxicity was calculated as cell viability $(\%)=$ sample OD/blank OD $\times 100$.

Statistical analysis: All data are expressed as mean \pm SD. Analysis was performed by one-way analysis of variance (ANOVA) followed by the Student-Newman-Keuls (S-N-K) test. Results were considered significantly different when $p$ values were less than 0.05 . Values bearing different superscripts indicate significant differences.

\section{RESULTS AND DISCUSSION}

Seven known compounds were isolated from stems of $Q$. acuta Thunberg. Structural elucidation of all the compounds was done with the help of 2-dimensional nuclear magnetic resonance (2D-NMR) and mass spectroscopy (MS) and the structure was confirmed by comparing the findings with those previously reported in the literature.

Extraction was based on solvent fractionation and was performed using hexane, ethyl acetate, butyl alcohol and water. The soluble fractions of ethyl acetate and butyl alcohol showed anti-oxidative activity with $\mathrm{IC}_{50}$ values of $18.09 \pm 1.53 \mu \mathrm{g} / \mathrm{mL}$ and $20.67 \pm 2.21 \mu \mathrm{g} / \mathrm{mL}$, respectively; the $\mathrm{IC}_{50}$ of L-ascorbic acid was found to be $4.29 \pm 0.08 \mu \mathrm{g} / \mathrm{mL}$ (data not shown). (+)-Catechin (1) $)^{13-15}$ and (-)-epicatechin $(\mathbf{2})^{4,16}$ were isolated from the butyl alcohol soluble fraction of the acetone extract. Taxifolin $(3)^{17,18}$, taxifolin 3- $O$ - $\beta$-D-glucopyranoside $(4)^{19,20}$, taxifolin $4^{\prime}-O-\beta$ D-glucopyranoside $(5)^{21,22}$, procyanidin B-3 $(6)^{23-25}$ and (+)- 
TABLE-1

ANTI-OXIDATIVE (DPPH RADICAL AND SUPEROXIDE ANION SCAVENGING ASSAYS) AND ANTI-INFLAMMATORY (INHIBITION OF NITRIC OXIDE PRODUCTION) ACTIVITIES OF COMPOUNDS 1-7. VALUES REPRESENT MEAN \pm SD OF THREE TRIALS. VALUES SUPERSCRIPTED DIFFERENTLY WITHIN A COLUMN ARE SIGNIFICANTLY DIFFERENT $(p<0.05)$

\begin{tabular}{cccc}
\hline Compound & $\begin{array}{c}\text { DPPH radical scavenging activity } \\
\mathrm{IC}_{50}(\mu \mathrm{M})\end{array}$ & $\begin{array}{c}\text { Superoxide anion scavenging } \\
\text { activity } \mathrm{IC}_{50}(\mu \mathrm{M})\end{array}$ & $\begin{array}{c}\text { Inhibition of NO production } \\
\mathrm{IC}_{50}(\mu \mathrm{M})\end{array}$ \\
\hline $\mathbf{1}$ & $37.92 \pm 1.23^{\mathrm{d}}$ & $40.52 \pm 2.15^{\mathrm{f}}$ & $62.01 \pm 1.42^{\mathrm{d}}$ \\
$\mathbf{2}$ & $26.37 \pm 1.72^{\mathrm{c}}$ & $29.91 \pm 2.53^{\mathrm{c}}$ & $72.56 \pm 1.99^{\mathrm{e}}$ \\
$\mathbf{3}$ & $72.48 \pm 2.41^{\mathrm{f}}$ & $39.35 \pm 4.39^{\mathrm{e}}$ & $20.32 \pm 1.57^{\mathrm{a}}$ \\
$\mathbf{4}$ & $>100^{\mathrm{h}}$ & $33.75 \pm 3.87^{\mathrm{d}}$ & $54.60 \pm 4.37^{\mathrm{c}}$ \\
$\mathbf{5}$ & $95.22 \pm 1.85^{\mathrm{g}}$ & $43.06 \pm 1.67^{\mathrm{f}}$ & $43.23 \pm 3.71^{\mathrm{c}}$ \\
$\mathbf{6}$ & $16.45 \pm 0.64^{\mathrm{a}}$ & $5.86 \pm 2.17^{\mathrm{b}}$ & $30.47 \pm 5.26^{\mathrm{b}}$ \\
$\mathbf{7}$ & $45.44 \pm 1.61^{\mathrm{e}}$ & $>100^{\mathrm{g}}$ & $15.50 \pm 0.84^{\mathrm{a}}$ \\
L-Ascorbic acid & $21.47 \pm 0.54^{\mathrm{b}}$ & - & - \\
Allopurinol & - & $2.53 \pm 0.04^{\mathrm{a}}$ & - \\
L-NMMA & - & - & $14.32 \pm 2.24^{\mathrm{a}}$ \\
\hline DPPH: 2, 2-diphenyl-1-picrylhydrazyl; NO: nitric oxide; L-NMMA: L- $N$-monomethylargininecitrate &
\end{tabular}

lyoniresinol $3 \alpha-O$ - $\beta$-D-xylopyranoside $(7)^{26,27}$ were isolated from the ethyl acetate soluble fraction (Fig. 1).

Our results suggest that phenolic compounds (1-7) could play an important role in the antioxidative and antiinflammatory effects of $Q$. acuta. Although all of these compounds have been reported previously, this is the first study to report the presence of phenolic compounds in $Q$. acuta. The data from general experimental procedures, ${ }^{1} \mathrm{H} \mathrm{NMR},{ }^{13} \mathrm{C} \mathrm{NMR}$ and MS, of compounds 1-7 are listed in the supplementary material.<smiles>Oc1cc(O)c2c(c1)O[C@H](c1ccc(O)c(O)c1)C(P)C2</smiles><smiles>Oc1cc(O)c2c(c1)O[C@H](c1ccc(O)c(O)c1)C(O)[C@H]2c1c(O)cc(O)c2c1O[C@H](c1ccc(O)c(O)c1)C(O)C2</smiles><smiles>CCOC1C(=O)c2c(O)cc(O)cc2OC1c1ccc(C(=O)O)c(O)c1</smiles><smiles>COc1cc(-c2c(COC3OC4C(O)C(O)C(O)C(O)C4O3)cc(OC)c3cc(OC)c(O)c(O)c23)cc(OC)c1O</smiles>

Fig. 1. Structures of compounds 1-7 isolated from $Q$. acuta

The antioxidative activities of the compounds of $Q$. acuta (1-7) were evaluated by DPPH free radical and superoxide dismutase (SOD)-like superoxide anion scavenging assays and expressed in terms of $\mathrm{IC}_{50}$ values $(\mu \mathrm{M})$. The $\mathrm{IC}_{50}$ values for the DPPH free radical scavenging activities of compounds $\mathbf{1 , 2}$ and 6 were $37.92 \pm 1.23,26.37 \pm 1.72$ and $16.45 \pm 0.64 \mu \mathrm{M}$, respectively. All compounds except 7 exhibited potent SODlike superoxide anion scavenging activities with $\mathrm{IC}_{50}$ values of $40.52 \pm 2.15,29.91 \pm 2.53,39.35 \pm 4.39,33.75 \pm 3.87$, $43.06 \pm 1.67$ and $5.86 \pm 2.17 \mu \mathrm{M}$ for compounds $\mathbf{1 - 6}$, respectively (Table-1). The antioxidant activity of $Q$. acuta may be because of the ortho-dihydroxy-phenolic moiety, which enables the transfer of a hydrogen atom to an active free radical $^{28}$.
The antiinflammatory activity of the constituents of $Q$. acuta (1-7) was evaluated by the extent of inhibition of NO production in LPS-induced RAW 264.7 cells and was expressed in terms of $\mathrm{IC}_{50}$ values $(\mu \mathrm{M})$. $\mathrm{IC}_{50}$ values for NO inhibition for compounds $\mathbf{3}, \mathbf{6}$ and 7 were $20.32 \pm 1.57,30.47 \pm 5.26$ and $15.50 \pm 0.84 \mu \mathrm{M}$, respectively (Table-1). Cytotoxicity of all compounds was measured by conducting the MTT assay, but there was no evidence of cytotoxicity at any of the concentrations studied. Therefore, compounds 3, 6 and 7 may have a role in the treatment of inflammation due to NO overproduction.

\section{Conclusion}

In this study, two flavan-3-ols (1 and $\mathbf{2})$, three flavanonols $(\mathbf{3}, 4$ and 5), one proanthocyanidin (6) and one neolignan (7) were isolated and identified from $Q$. acuta. 1, 2 and $\mathbf{6}$ showed strong antioxidative activities. Further, compounds 3, 6 and $\mathbf{7}$ showed strong antiinflammatory activities. Therefore, these compounds present in $Q$. acuta can greatly benefit humans because of their antioxidative and antiinflammatory effects.

\section{ACKNOWLEDGEMENTS}

This research was supported by Basic Science Research Program through the National Research Foundation of Korea (NRF) funded by the Ministry of Education, Science and Technology (2010-0022929).

\section{REFERENCES}

1. Y.N. Lee, Flora of Korea. Seoul, Republic of Korea: Kyo-Hak Publishing (1996).

2. K.S. Ko, E.S. Jeon, Ferns, Fern-Allies and Seed-Bearing Plants of Korea, Il-Jin-Sa Publishing, Seoul, Republic of Korea (2003).

3. G. Nonaka, H. Nishimura and I. Nishioka, Chem. Pharm. Bull. (Tokyo), 30, 2061 (1982).

4. G. Nonaka and I. Nishioka, Chem. Pharm. Bull. (Tokyo), 30, 4268 (1982).

5. G. Nonaka, H. Nishimura, I. Nishioka, J. Chem. Soc., Perkin Trans. I, 159 (1985)

6. G. Nonaka, T. Sakai, T. Tanaka, K. Mihashi and I. Nishioka, Chem. Pharm. Bull. (Tokyo), 38, 2151 (1990).

7. M. Serit, T. Okubo, R.H. Su, N. Hagiwara, M. Kim, T. Iwagawa and T. Yamamoto, Agric. Biol. Chem., 55, 19 (1991).

8. T. Hatano, R. Edamatsu, M. Hiramatsu, A. Mori, Y. Fujita and T. Yasuhara, Chem. Pharm. Bull. (Tokyo), 37, 2016 (1989).

9. I. Parejo, F. Viladomat, J. Bastida, A. Rosas-Romero, N. Flerlage, J. Burillo and C. Codina, J. Agric. Food Chem., 50, 6882 (2002). 
10. T. Mosmann, J. Immunol. Methods, 65, 55 (1983).

11. B. Epe, D. Ballmaier, I. Roussyn, K. Brivida and H. Sied, Nucleic Acids Res., 24, 4105 (1996).

12. S.Y. Park, S.S. Hong, X.H. Han, J.S. Ro and B.Y. Hwang, Nat. Prod. Sci., 11, 85 (2005).

13. Y. Lu and L. Yeap Foo, Food Chem., 65, 1 (1999).

14. Y. Sudjaroen, R. Haubner, G. Würtele, W.E. Hull, G. Erben, B. Spiegelhalder, S. Changbumrung, H. Bartsch and R.W. Owen, Food Chem. Toxicol., 43, 1673 (2005).

15. M. Watanabe, J. Agric. Food Chem., 46, 839 (1998).

16. D.S. Yang, W.K. Whang and I.H. Kim, Arch. Pharm. Res., 19, 507 (1996).

17. R. Zerback, M. Bokel, H. Geiger and D. Hess, Phytochemistry, 28, 897 (1989).

18. J.H. Lee, W.J. Jeon, E.S. Yoo, C.M. Kim and Y.S. Kwon, Nat. Prod. Sci., 11, (2005).
19. Z. Shen and O. Theander, Phytochemistry, 24, 155 (1985).

20. S.E. Choi, K.H. Park, B.H. Han, M.S. Jeong, S.J. Seo, D.I. Lee, S.S. Joo and M.W. Lee, Phytother. Res., 25, (2011).

21. T. Fossen, A.T. Pedersen and O.M. Andersen, Phytochemistry, 47, 281 (1998).

22. X. Xu, H. Xie, J. Hao, Y. Jiang and X. Wei, J. Agric. Food Chem., 59, 1205 (2011).

23. M.W. Lee, T. Tanaka, G.I. Nonaka and D.R. Hahn, Arch. Pharm. Res., 15, 211 (1992).

24. A. Benavides, P. Montoro, C. Bassarello, S. Piacente and C. Pizza, J. Pharm. Biomed. Anal., 40, 639 (2006).

25. J. Fan, X. Ding and W. Gu, Food Chem., 102, 168 (2007)

26. E. Smite, P. Hefeng and L.N. Lundgren, Phytochemistry, 40, 341 (2001).

27. T. Kanchanapoom, R. Kasai and K. Yamasaki, Phytochemistry, 57, 1245 (2011).

28. J. Han, X. Weng and K. Bi, Food Chem., 106, 2 (2008). 